\title{
Subaru optical observations of the two middle-aged pulsars PSR B0656+14 and Geminga ${ }^{\star}$
}

\author{
Y. A. Shibanov ${ }^{1}$, S. V. Zharikov ${ }^{2}$, V. N. Komarova ${ }^{3,4}$, N. Kawai ${ }^{5}$, Y. Urata ${ }^{6}$, \\ A. B. Koptsevich ${ }^{1,7}$, V. V. Sokolov ${ }^{3,4}$, S. Shibata $^{8}$, and N. Shibazaki ${ }^{9}$ \\ ${ }^{1}$ Ioffe Physical Technical Institute, Politekhnicheskaya 26, St. Petersburg 194021, Russia \\ e-mail: shib@astro.ioffe.ru \\ 2 Observatorio Astronomico Nacional SPM, Instituto de Astronomia, UNAM, Ensenada, BC, Mexico \\ 3 Special Astrophysical Observatory of RAS, Karachai-Cherkessia, Nizhnij Arkhyz 369167, Russia \\ ${ }^{4}$ Isaac Newton Institute of Chile, SAO Branch, Russia \\ 5 Department of Physics, Tokyo Institute of Technology, 2-12-1 Ookayama, Muguro-ku, Tokyo 152-8551, Japan \\ ${ }^{6}$ RIKEN (Institute of Physical and Chemical Research) Hirosawa, Wako, Saitama 351-0198, Japan \\ 7 University of Toronto, 60 St George St, Toronto, ON M5S1A7, Canada \\ 8 Yamagata University, Yamagata 990-8560, Japan \\ 9 Rikkyo University, Tokyo 171-8501, Japan
}

Received 8 September 2005 / Accepted 17 October 2005

\section{ABSTRACT}

\begin{abstract}
Aims. We carried out a deep subarcsecond BRI imaging of the two middle-aged pulsars to establish their properties in the optical range. Methods. Astrometry and photometry methods are applied to identify the pulsars and to measure their fluxes. We also reanalyze archival ESO/NTT and HST broadband data and find that some published fluxes for Geminga were estimated inaccurately. The resulting dereddened broadband spectra in the near-IR-UV range are analyzed and compared with available data from the radio through gamma-rays.

Results. Both pulsars are detected at $\gtrsim 10 \sigma$ level. Geminga is for the first time reliably detected in the $I$ band with a magnitude of 25 m $10 \pm 0.14$. The dereddened spectra of both pulsars are remarkably similar to each other and show significant flux increases towards the far-UV and near-IR, and a wide flux excess in $V-I$ bands. This suggests a multicomponent structure of the optical emission. The nonthermal power law component of the pulsar magnetospheric origin dominates in the most part of the optical range. For PSR B0656+14 it is compatible with a low energy extension of the power law tail seen in hard X-rays. For Geminga the respective extension overshoots by a factor of 100 the nonthermal optical flux, which has a less steep spectral slope than in X-rays. This implies a spectral break at a photon energy $\sim 1 \mathrm{keV}$. The flux increases towards the far-UV are compatible with contributions of the Rayleigh-Jeans parts of the blackbody components from whole surfaces of the neutron stars dominating in soft X-rays. The $V-I$ excess, which is most significant for PSR B0656+14, suggests a third spectral component of still unidentified origin. Faint, a few arcseconds in size nebulae extended perpendicular to the proper motion directions of the pulsars, are seen around both objects in our deepest $I$ band images. They can be optical counterparts of the bow-shock head of Geminga and of the tentative pulsar wind nebula of PSR B0656+14 observed in X-rays.
\end{abstract}

Key words. pulsars: individual: Geminga (PSR J0633+1746), PSR B0656+14 (PSR J0659+1414) - stars: neutron pulsars: general - radiation mechanisms: general

\section{Introduction}

The two nearby middle-aged pulsars, PSR B0656+14 and Geminga (PSR J0633+1746), belong to a small group of rotation powered isolated neutron stars (NSs) whose emission is detected in a wide spectral range spanning from radio through gamma-rays. Their parameters, such as the period $P$ and its derivative $\dot{P}$, characteristic age $\tau$, spin-down luminosity $\dot{E}$,

^ Based on data collected at Subaru Telescope, which is operated by the National Astronomical Observatory of Japan. magnetic field $B$, are very similar (Table 1). This is reflected in similarities of observational manifestations of both pulsars.

Multiwavelength spectra of PSR B0656+14 and Geminga consist of two main components. The first one is a nonthermal component which is believed to be generated in magnetospheres of the NSs. It dominates the pulsar emission in almost the whole observed range and its spectrum is described by a power law, albeit with different spectral indices in different spectral domains. Multiwavelength properties of this component are important for the study of not yet clearly understood emission mechanisms in magnetospheres of NSs. The second 
Table 1. Parameters of Geminga and PSR B0656+14 (from Taylor et al. 1993, unless specified otherwise).

\begin{tabular}{|c|c|c|}
\hline & Geminga & B $0656+14$ \\
\hline \multicolumn{3}{|l|}{ Observed } \\
\hline$\alpha(\mathrm{J} 2000)^{a} \ldots$ & $6^{\mathrm{h}} 33^{\mathrm{m}} 54^{\mathrm{s}} .1530(28)^{b}$ & $6^{\mathrm{h}} 59^{\mathrm{m}} 48^{\mathrm{s}} .1472(7)^{c}$ \\
\hline$\delta(\mathrm{J} 2000)^{a} \ldots$ & $17^{\circ} 46^{\prime} 12^{\prime \prime} \cdot 909(40)^{b}$ & $14^{\circ} 14^{\prime} 21^{\prime \prime} .160(10)^{c}$ \\
\hline$\mu_{\alpha}^{a}\left(\operatorname{mas~yr}^{-1}\right)$. & $138(4)^{d}$ & $45.47(65)^{c}$ \\
\hline$\mu_{\delta}^{a}\left(\operatorname{mas~yr}^{-1}\right)$ & $97(4)^{d}$ & $-2.40(29)^{c}$ \\
\hline$\pi^{a}(\operatorname{mas}) \ldots \ldots$ & $6.36(1.74)^{d}$ & $3.47(36)^{c}$ \\
\hline$l^{e} \ldots \ldots \ldots$ & $195^{\circ} .134$ & $201: 108$ \\
\hline$b^{e}$ & 4.265 & 8.258 \\
\hline$P(\mathrm{~ms}) \ldots \ldots$ & 237 & 385 \\
\hline$\dot{P}\left(10^{-15}\right) \ldots$ & 10.97 & 55.01 \\
\hline$D M^{f}\left(\mathrm{~cm}^{-3} \mathrm{pc}\right)$ & $2.9^{g}$ & 14.02 \\
\hline \multicolumn{3}{|l|}{ Derived } \\
\hline$d^{a}(\mathrm{pc}) \ldots \ldots$ & $157\left(\begin{array}{c}+59 \\
-34\end{array}\right)^{d}$ & $288\left({ }_{-27}^{+33}\right)^{c}$ \\
\hline$\tau^{h}(\mathrm{kyr}) \ldots \ldots$ & 342 & 111 \\
\hline $\log B(\mathrm{G}) \ldots$ & 12.21 & 12.67 \\
\hline $\log \dot{E}\left(\operatorname{erg~s}^{-1}\right)$ & 34.57 & 34.58 \\
\hline
\end{tabular}

${ }^{a}$ Coordinates, proper motion, parallax, and parallax-based distance; hereafter the numerals in parentheses are $1 \sigma$ uncertainties referring to last significant digits quoted; ${ }^{b}$ Caraveo et al. (1998) (epoch 1995.21); ${ }^{c}$ Brisken et al. (2003); ${ }^{d}$ Caraveo et al. (1996); ${ }^{e}$ galactic coordinates; ${ }^{f}$ dispersion measure; ${ }^{g}$ Malofeev \& Malov (1997); ${ }^{h}$ characteristic age $P / 2 \dot{P}$.

component dominates in soft X-ray and EUV ranges where it is seen as a strong excess over the nonthermal background. It is well described by a blackbody spectrum and this excess is thought to be associated with thermal emission form cooling surfaces of the NSs (Becker \& Trümper 1997; Zavlin \& Pavlov 2004a; De Luca et al. 2005). The study of this component is of a crucial importance to evaluate the NS surface temperature, to compare it with NS cooling theories and to obtain information on still poorly known properties and equation of state of super-dense matter in interiors of NSs (e.g., Yakovlev $\&$ Pethick 2004). Inclusion of an additional high temperature thermal component assumed to be originated from hot pulsar polar caps appears to improve significantly spectral fits in X-rays (Koptsevich et al. 2001; Pavlov et al. 2002; Zavlin \& Pavlov 2004a; De Luca et al. 2005).

There are, however, noticeable differences between the two objects. Geminga is known as one of the brightest gammaray sources on the sky (Bignami \& Caraveo 1996), while PSR B0656+14 is fainter and only marginally detected in gamma-rays (Ramanamurthy et al. 1996). In the radio range PSR B0656+14 is a stable source with properties to be typical for an ordinary radio pulsar. In contrast, Geminga has been considered for a long time as a "radio-quiet" NS, until it was detected at rather low (only $\lesssim 102.5 \mathrm{MHz}$ ) frequencies (Kuz'min \& Losovskii 1997; Malofeev \& Malov 1997; Shitov \& Pugachev 1998). Geminga has unusually steep radio spectrum
(Burderi et al. 1999), and unlike most of radio pulsars its pulse profile is very unstable (Kassim \& Lazio 1999).

PSR B0656+14 has a twice lower transverse velocity, $v_{\perp} \approx 60 \mathrm{~km} \mathrm{~s}^{-1}$ (Mignani et al. 2000; Brisken et al. 2003), than Geminga, $v_{\perp} \approx 122 \mathrm{~km} \mathrm{~s}^{-1}$ (Caraveo et al. 1996), and both pulsars move in different environment, as indicated by their dispersion measure values (cf. Table 1). PSR B0656+14 sits near the center of the Monogem ring (Nousek et al. 1981), that is a bright $\sim 10^{5} \mathrm{yr}$ expanding supernova remnant (SNR) likely produced at the same supernova explosion as the pulsar (Thorsett et al. 2003). Geminga has not been associated with any known SNR, although based on its proper motion and age a birth place was proposed to be located inside the Cas-Tau OB or the Ori OB1a stellar associations (Pellizza et al. 2005). Recent XMM-Newton observations of this pulsar in X-rays revealed a faint bow-shock structure whose tails extend up to $2^{\prime}$ from the pulsar and are elongated along direction of its proper motion (Caraveo et al. 2003). The structure has not yet been identified in the optical range. No reliable signs of a bow-shock nebula have been detected around more distant and slowly moving PSR B0656+14. Some hints of a compact, $3^{\prime \prime}-4$ ", faint pulsar wind nebula (PWN) surrounding the pulsar were reported based on the X-ray images obtained with the Chandra/LETG/HRC-S (Marshall \& Schulz 2002) and with the Chandra/ACIS-S3 (Pavlov et al. 2002), but they have not yet been confirmed by deeper exposures and have not been identified in other spectral domains.

Both pulsars are firmly identified in the optical range by an accurate positional coincidence of the optical counterparts with the pulsars, by comparison of the proper motion in radio and optical ranges (Caraveo et al. 1996; Mignani et al. 2000; Brisken et al. 2003), and by detection of pulsations with the pulsar period in the optical (PSR B0656+14: Shearer et al. 1997; Kern et al. 2003. Geminga: Shearer et al. 1998) and in UV (PSR B0656+14: Shibanov et al. 2005; Geminga: Kargaltsev et al. 2005).

Due to faintness of the pulsar counterparts the spectral information in the optical is restricted mainly by the broadband photometry. Published spectral observations of Geminga (Martin et al. 1998) are too noisy to compete with the broadband data. The broadband far-UV (FUV) spectra of both pulsars appear to be dominated by the Rayleigh-Jeans (RJ) part of the blackbody spectrum extrapolated from soft X-rays (Mignani et al. 1998; Koptsevich et al. 2001, hereafter K2001). For Geminga this is supported also by FUV spectral data recently reported by Kargaltsev et al. (2005). At longer wavelengths there is a significant flux excess over the RJ extrapolation in the spectra of both pulsars.

For each of the pulsars these excesses have been interpreted in a different way. For PSR B0656+14 the spectral energy distribution from the optical to near-IR (NIR) ranges was shown to be compatible with the low energy extension of a high energy nonthermal spectral tail detected in X-rays (K2001). It suggested possible common origin of the nonthermal spectral component in the optical and X-rays. Early attempts of detection of Geminga in NIR were unsuccessful and the measured upper limit in the $I$ band suggested a strong spectral dip in this band followed by a prominent excess in the 
$V$ band (Mignani et al. 1998). The shape of the excess reminded a broad emission feature over the RJ continuum that has been interpreted as an ion cyclotron emission from a hot non-uniformly magnetized plasma covering the pulsar polar caps (Jacchia et al. 1999). However, later Geminga was detected in the NIR (Harlow et al. 1998). Preliminary data analysis (K2001) revealed a flux growth towards longer wavelengths, as it is in case of PSR B0656+14. This does not quite agree with the cyclotron interpretation. In addition, Kurt et al. (2001) reported on a tentative detection of Geminga in the $I$ band at a flux level, which is by a half order of magnitude higher than the upper limit known previously. The reported flux value is compatible with a smooth connection between the $V$ and NIR bands, that has made the cyclotron interpretation even more questionable.

In any case, published broadband optical data are still too uncertain to reject or confirm surely the suggested interpretations and to understand whether these two middle-aged pulsars have similar or different properties in the optical range. The uncertainties do not allow us to study also significance of the spectral difference between the middle-aged and other pulsars of different ages detected in optical range (K2001; Zharikov et al. 2002, 2003; Shibanov et al. 2003). The main source of the flux errors in case of PSR B0656+14 is a contamination of the pulsar flux by a background extended object in $\simeq 1$ ". 1 from the pulsar (K2001). For about twice fainter Geminga, there are no contaminating objects but deeper exposures are necessary to establish its optical spectral energy distribution with a higher accuracy.

In this paper we report on a subarcsecond deep broadband imaging of the Geminga and PSR B0656+14 fields with the Subaru telescope. Preliminary results have been reported by Komarova et al. (2003). Here we analyze our data together with the available NIR-optical-UV data from the HST, ESO/NTT, and $6 \mathrm{~m}$ BTA telescope, as well as with available multiwavelength data from the radio through gamma-rays. In Sect. 2 we describe the observations, data reduction, astrometric and photometric referencing. The results are presented in Sect. 3 and discussed in Sect. 4.

\section{Observations and data analysis}

\subsection{Subaru observations and data reduction}

Two fields containing Geminga and PSR B0656+14 were observed on January 21, 2001 with the wide field camera Suprime-Cam at the primary focus of the Subaru telescope. The Suprime-Cam (Miyazaki et al. 1998) was equipped with ten MIT/LL $2048 \times 4096$ CCDs arranged in a $5 \times 2$ pattern to provide $34^{\prime} \times 27^{\prime}$ FOV with the pixel size on the sky of $0.201^{\prime \prime} \times 0.201^{\prime \prime}$. The pulsars were exposed in one of the CCD chips, si006s, with the Gain $=1.17$, making use of the $B, R$, and $I$ filters with throughput to be close to the Johnson-Cousins system. Sets of 5-10 min individual dithered exposures were obtained in each of the filters to get rid of cosmic rays at further data analysis. Several individual exposures were removed from the consideration due to problems with the telescope guiding system. Thus the total exposure time used for the subsequent
Table 2. Log of observations of Geminga and PSR B0656+14 with the Subaru.

\begin{tabular}{|c|c|c|c|c|c|}
\hline PSR & $\begin{array}{l}\text { UT } \\
21 \text { Jan. } 2001\end{array}$ & $\begin{array}{l}\text { Exp. } \\
\text { s }\end{array}$ & Band & $\begin{array}{l}\text { Seeing } \\
\operatorname{arcsec}^{a}\end{array}$ & $\operatorname{Sec} z$ \\
\hline Geminga & $05: 17: 26.9$ & 332.3 & $I$ & 0.66 & 1.56 \\
\hline Geminga & $05: 30: 12.6$ & 300.0 & $I$ & 0.66 & 1.47 \\
\hline Geminga & $05: 42: 29.2$ & 300.0 & $I$ & 0.70 & 1.39 \\
\hline Geminga & $05: 51: 53.6$ & 300.0 & $I$ & 0.65 & 1.35 \\
\hline Geminga & $06: 01: 30.5$ & 300.0 & $I$ & 0.68 & 1.30 \\
\hline Geminga & $06: 31: 27.7$ & 300.0 & $I$ & 0.55 & 1.19 \\
\hline Geminga & $06: 40: 32.8$ & 300.0 & $I$ & 0.55 & 1.16 \\
\hline Geminga & $06: 49: 38.0$ & 400.0 & $I$ & 0.55 & 1.13 \\
\hline Geminga & $07: 00: 22.8$ & 400.0 & $I$ & 0.60 & 1.11 \\
\hline Geminga & 07:11:07.8 & 400.0 & $I$ & 0.58 & 1.09 \\
\hline Geminga & $07: 21: 52.8$ & 400.0 & $I$ & 0.60 & 1.07 \\
\hline Geminga & $07: 32: 38.2$ & 400.0 & $I$ & 0.60 & 1.05 \\
\hline Geminga & $07: 43: 23.9$ & 400.0 & $I$ & 0.60 & 1.04 \\
\hline Geminga & 08:07:55.3 & 400.0 & $I$ & 0.61 & 1.01 \\
\hline Geminga & $08: 26: 36.9$ & 400.0 & $I$ & 0.62 & 1.00 \\
\hline B0656+14 & $08: 46: 10.7$ & 400.0 & $I$ & 0.65 & 1.01 \\
\hline B $0656+14$ & $08: 56: 55.8$ & 400.0 & $I$ & 0.65 & 1.01 \\
\hline B $0656+14$ & 09:07:40.9 & 400.0 & $I$ & 0.73 & 1.00 \\
\hline B $0656+14$ & $09: 18: 26.0$ & 400.0 & $I$ & 0.67 & 1.00 \\
\hline B $0656+14$ & 09:29:10.9 & 400.0 & $I$ & 0.65 & 1.01 \\
\hline B0656+14 & 09:39:55.9 & 400.0 & $I$ & 0.63 & 1.01 \\
\hline B0656+14 & $10: 22: 05.8$ & 510.0 & $R$ & 0.60 & 1.04 \\
\hline B $0656+14$ & $10: 34: 41.0$ & 510.0 & $R$ & 0.60 & 1.06 \\
\hline Geminga & $10: 47: 15.8$ & 510.0 & $R$ & 0.61 & 1.13 \\
\hline Geminga & $10: 59: 58.6$ & 510.0 & $R$ & 0.68 & 1.17 \\
\hline Geminga & $11: 12: 33.8$ & 510.0 & $R$ & 0.61 & 1.21 \\
\hline Geminga & $11: 25: 09.0$ & 510.0 & $R$ & 0.60 & 1.25 \\
\hline B $0656+14$ & $11: 54: 36.9$ & 600.0 & $B$ & 0.65 & 1.28 \\
\hline B $0656+14$ & $12: 08: 42.3$ & 600.0 & $B$ & 0.66 & 1.35 \\
\hline B0656+14 & $12: 22: 47.8$ & 600.0 & $B$ & 0.69 & 1.43 \\
\hline
\end{tabular}

${ }^{a}$ FWHM of the stellar profile in the individual images.

analysis of Geminga was $2040 \mathrm{~s}$ in the $R$ and $5332.3 \mathrm{~s}$ in the $I$ bands. For a brighter PSR B0656+14 it was $1800 \mathrm{~s}, 1020 \mathrm{~s}$, and $2400 \mathrm{~s}$ in the $B, R$, and $I$ bands, respectively. The observational conditions were rather stable during the night with seeing varying from $\sim 0$ ' $^{\prime} 6$ to $\sim 0$.' 7 . A Log of observations is given in Table 2. The PG1047+003 standard field of Landolt (1992) used for the photometric calibration was observed at the end of the night.

Standard data reduction including bias subtraction and flatfielding was performed making use of the MIDAS and IRAF software. In order to get rid of the remaining cosmic rays and to decrease possible contamination of PSR B0656+14 by a nearby extended object we combined the individual dither images of the pulsar field in the $B$ and $I$ bands making use of the ditherII package by Fruchter et al. 1998.

\subsection{Astrometric referencing of the Subaru images}

The positions and proper motions of Geminga and PSR B0656+14 are well established (see Table 1). To 
Table 3. Positions of Geminga and PSR B0656+14 at the epoch of the Subaru observations, 2001.054.

\begin{tabular}{lllll}
\hline \hline \multicolumn{1}{c}{ Object } & \multicolumn{2}{c}{ Expected } & \multicolumn{2}{c}{ Observed } \\
& \multicolumn{1}{c}{$\alpha_{2000}$} & \multicolumn{1}{c}{$\delta_{2000}$} & $\alpha_{2000}$ & $\delta_{2000}$ \\
\hline Geminga & $06^{\mathrm{h}} 33^{\mathrm{m}} 54.2067(32)$ & $17^{\circ} 46^{\prime} 13^{\prime \prime} \cdot 475(46)$ & $06^{\mathrm{h}} 33^{\mathrm{m}} 54^{\mathrm{s}} .219(15)$ & $17^{\circ} 46^{\prime} 13^{\prime \prime} \cdot 56(22)$ \\
PSR B0656+14 & $06^{\mathrm{h}} 59^{\mathrm{m}} 48.1503(7)$ & $14^{\circ} 14^{\prime} 21^{\prime \prime} \cdot 1575(10)$ & $06^{\mathrm{h}} 59^{\mathrm{m}} 48^{\mathrm{s}} .152(14)$ & $14^{\circ} 14^{\prime} 21^{\prime \prime} \cdot 41(21)$ \\
\hline
\end{tabular}
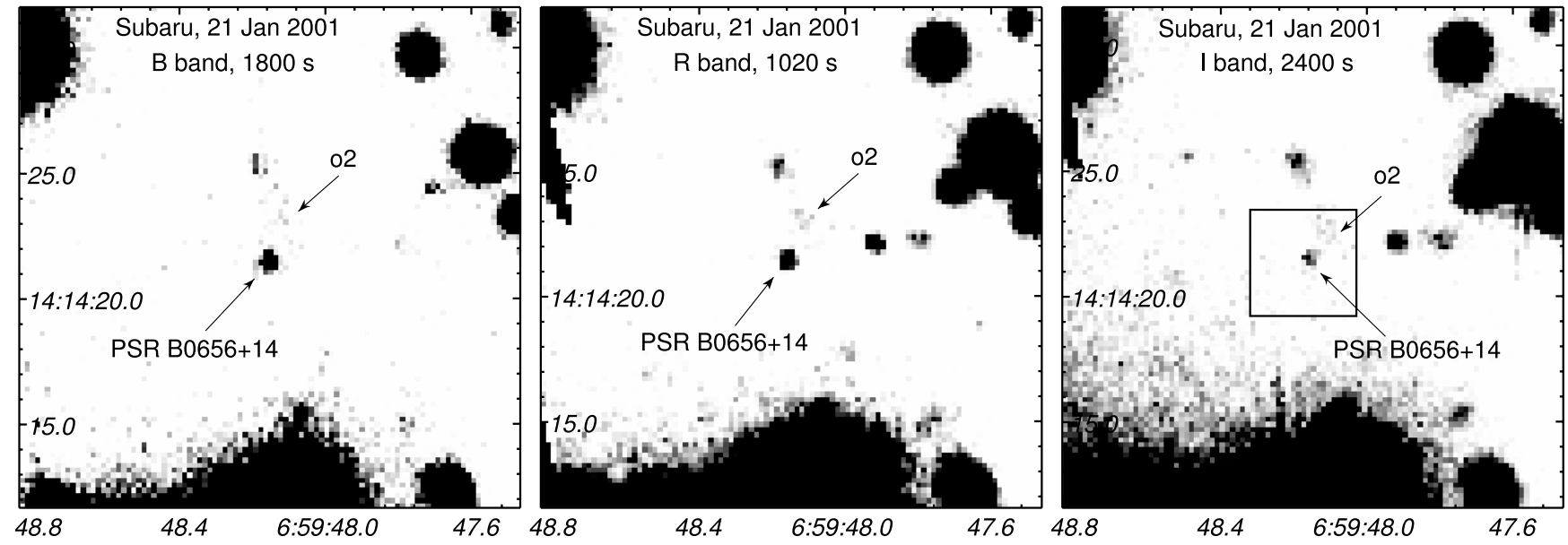

Fig. 1. $20^{\prime \prime} \times 20^{\prime \prime}$ fragments of the Subaru images of the PSR B0656+14 field in the $B, R$, and $I$ bands. Units of RA (horizontal axis) are hours, minutes and seconds, and units of Dec (vertical one) are degrees, arcminutes and arcseconds. A box in the right panel marks the region which is magnified in Fig. 6 and discussed in Sect. 3.2.

determine the expected coordinates of Geminga at the epoch of the Subaru observations (2001.054) its position at the epoch of the observations with HST/WFPC2 (1995.21) (Caraveo et al. 1996) was used as a reference point and then corrected for the proper motion. The same was done for PSR B0656+14 using a reference point from Brisken et al. (2003). based on the radio observations with the VLBA. The expected pulsar coordinates are given in Table 3 with errors accounting for the proper motion and reference point uncertainties.

The astrometric referencing of the Subaru images was done making use of the USNO-B1.0 catalog (Monet et al. 2003) and IRAF tasks ccmap/cctran. For the astrometric transformation of the combined images of the Geminga field we used the positions of six reference $\operatorname{stars}^{1}$. Formal rms errors of the astrometric fit for the RA and Dec were $\approx 0$.'07 and $\approx 0$.'06, respectively, for both the $R$ and $I$ images. Seven reference stars ${ }^{2}$ were used in case of PSR B0656+14. For each of the $B, R$, and $I$ images formal rms errors were $\approx 0$. $^{\prime} 08$ and $\approx 0$. $^{\prime} 05$, for the RA and Dec, respectively, which is less than the nominal USNO-B1.0 catalogue accuracy of 0.20 . Combining the rms errors and the catalog uncertainty we estimate the total $1 \sigma$ accuracy of our astrometric referencing of all the images as 0.21 in both RA and Dec. The optical counterparts to both pulsars are clearly seen at the expected positions (see Figs. 1 and 2). The expected and

1 USNO-B1.0 stars used for the astrometric transformation of the Geminga field: 1077-0137329, 1078-0139943, 1077-0137377, 10780139792, 1078-0139780 and 1077-0137166.

2 USNO-B1.0 stars used for the transformation of the PSR B0656+14 field: 1042-0123510, 1042-0123514, 1042-0123581, 1042-0123600, 1042-0123481, 1042-0123424 and 1042-0123352.

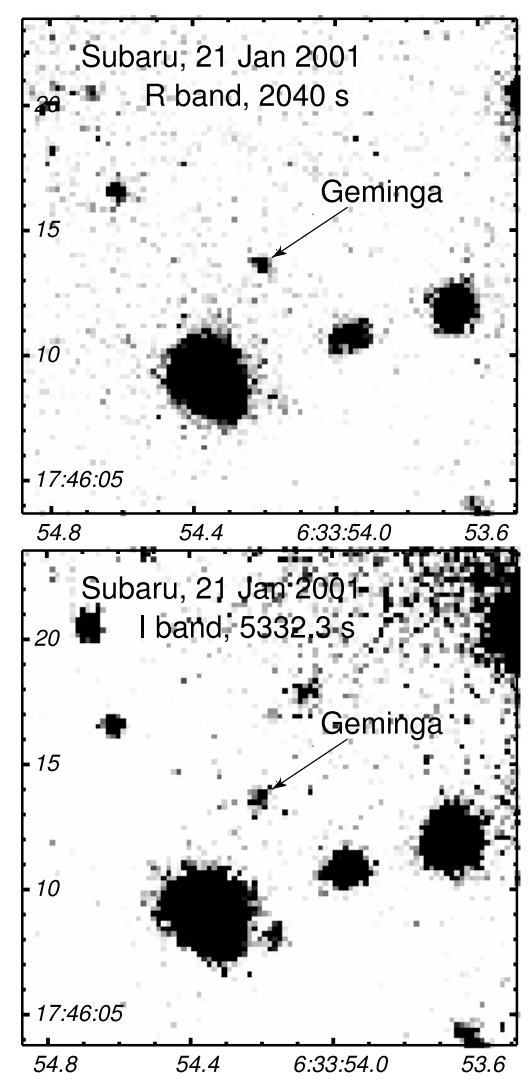

Fig. 2. $20^{\prime \prime} \times 20^{\prime \prime}$ fragments of the Subaru images of the Geminga field in the $R$ (top) and $I$ (bottom) bands. Axes orientation and label notations are the same as in Fig. 1. 

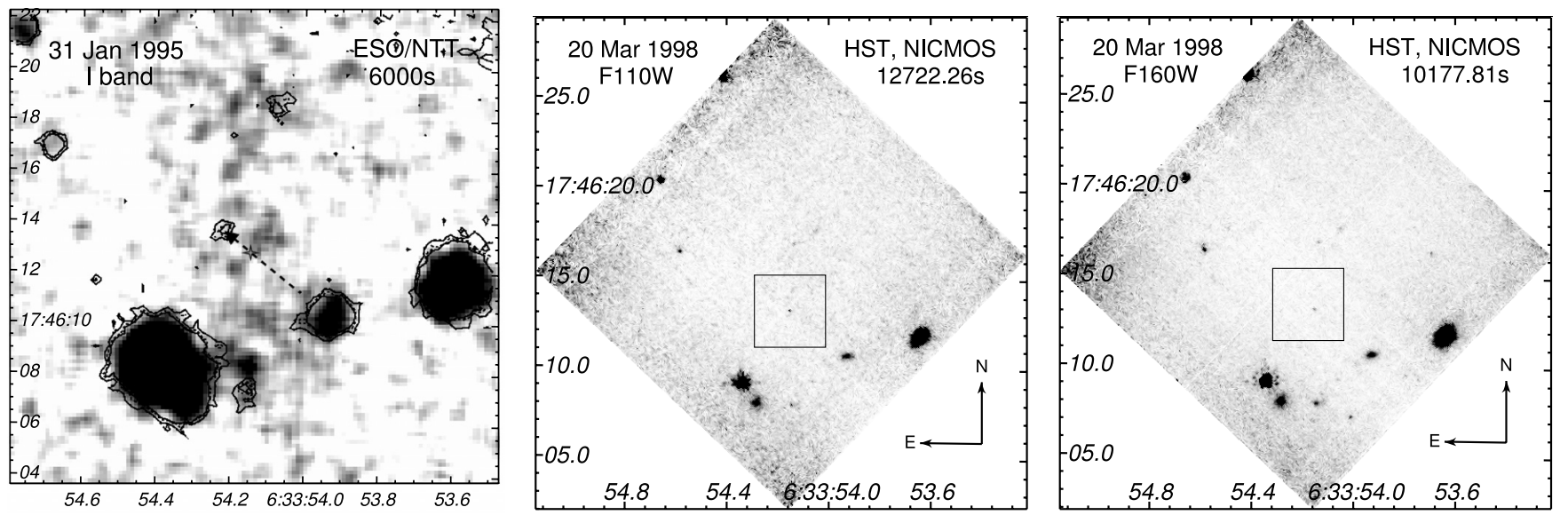

Fig. 3. Left: a fragment of the Geminga field image obtained with the ESO/NTT in the $I$ band (Bignami et al. 1996). The contours of the Subaru $I$ band image are overlaid and the dashed-line arrow shows the direction of the pulsar motion; the cross marks the position of the pulsar at the epoch of the ESO/NTT observations. Middle and right: near-infrared images of the same field obtained with the HST/NICMOS in the F110W and $F 160 \mathrm{~W}$ bands, respectively (Harlow et al. 1998). Boxes mark regions with Geminga, they are magnified in Fig. 4.

observed positions (Table 3) coincide with each other within the errors accounting for the centroid and astrometric referencing uncertainties.

\subsection{Photometric referencing of the Subaru images}

Photometric calibration was carried out using three standard stars from the Landolt field PG1047+003 (Landolt 1992), observed the same night and five secondary standard stars from the PSR B0656+14 field (Kurt et al. 1998). The atmospheric extinction factors in the $B, R$, and $I$ bands were derived from the variation of the count rates of field stars with the airmass changing during our observations (see Table 2). The values obtained $k_{B}=0 .{ }^{\mathrm{m}} 18 \pm 0.02, k_{R}=0.117 \pm 0.021, k_{I}=0.042 \pm$ 0.020 are consistent with the mean values at the Subaru site (Y. Komiyama, private communication). Insignificant decrease of the extinction, within $1 \sigma$ level, was noticed from the beginning to the end of observations. Based that the following transition equations related to the observational night were obtained:

$$
\begin{aligned}
B-b & =28.283(20)+0.094(10) \cdot(b-r) \\
R-r & =28.122(29)-0.023(14) \cdot(b-r) \\
R-r & =28.070(44)-0.138(47) \cdot(r-i) \\
I-i & =27.340(38)-0.152(55) \cdot(r-i)
\end{aligned}
$$

where $b, r, i$ are instrumental magnitudes and $B, R, I$ are magnitudes in the Johnson-Cousins photometric system. The errors of the transformation coefficients shown in parentheses include the extinction factor and standard instrumental magnitude uncertainties.

\subsection{The data from the ESO/NTT and HST archives}

In our analysis we used also the $I$-band images of the Geminga field obtained with the ESO/NTT (Bignami et al. 1996), kindly provided by Mignani. Five 20 min science exposures were obtained on January 31, 1995 using the SUSI ${ }^{3}$. The reduction of these data was rather complicated due to the absence of a standard "flatfield" image in the archive. To produce an artificial flatfield we subtracted stars from each science exposure and combined these images into a median one. It was slightly improved in the regions around the star positions making use of the MODIFY/AREA task of the ESO/MIDAS package, smoothed with the $5 \times 5$ pixel average and used as the flatfield in a standard data reduction. The problems with the flatfield and the fact that the individual science images were taken without shifts enough to remove accurately all artifacts led to the appearance of artificial extended objects around the position of Geminga in the final image (Fig. 3, left panel), precluding from a reliable identification of the pulsar (see Sect. 3.1. for details).

The NIR-optical-UV data from the HST archive ${ }^{4}$, obtained with the HST/NICMOS in the F110W and F160W bands (HST analogs of $J$ and $H$; Harlow et al. 1998) and with the HST/WFPC2 in the $F 555 \mathrm{~W}$ band for Geminga, with the HST/FOC in the F195W, F342W, F430W bands for Geminga (Bignami et al. 1996; Mignani et al. 1998) and for PSR B0656+14 (Pavlov et al. 1996, 1997) were also reanalysed. The data reduction and analysis of the NICMOS images obtained at the epoch of 1998.22 were performed as described in $\mathrm{K} 2001^{5}$. The photometry of the WFPC2 images processed by pipeline was performed using the algorithm described in the WFPC2 Data analysis Tutorial ${ }^{6}$. The reduction of the FOC images was done in the same manner as described in Pavlov et al. $(1996,1997)$ making use of the FOC Instrument Handbook (v6)

\footnotetext{
${ }^{3}$ Observational conditions and other details can be found in Bignami et al. (1996).

${ }^{4}$ archive.stsci.edu

5 See also the HST Data Handbook for NICMOS, stsci.edu/hst/nicmos/documents/handbooks/

${ }^{6}$ Baggett et al. (2002), in HST WFPC2 Data Handbook, v4, ed. B. Mobasher, Baltimore, STScI.

7 stsci.edu/ftp/instrument news/FOC/Foc handbook
} 
Table 4. Flux measurements of the pulsar optical counterparts (see Sect. 2.3 for details).

\begin{tabular}{|c|c|c|c|c|c|c|c|c|c|}
\hline Object & Band & $\begin{array}{l}\text { Seeing }^{a} \\
\text { pix }\end{array}$ & $S / N$ & $\begin{array}{l}d^{b} \\
\text { pix }\end{array}$ & $\begin{array}{c}\text { Source }^{c} \\
-2.5 \log (\text { counts } / \mathrm{s})\end{array}$ & $\begin{array}{l}k / \cos (Z)^{d} \\
\operatorname{mag}\end{array}$ & $\begin{array}{l}\delta_{\text {fin }} d^{e} \\
\text { mag }\end{array}$ & $\operatorname{Mag}^{f}$ & $\begin{array}{l}\text { Flux } \\
\mu \mathrm{Jy}\end{array}$ \\
\hline \multirow[t]{3}{*}{ PSR B0656+14 } & $B$ & 4.5 & 22 & 4 & -1.68 & 0.25 & 1.12 & $25.27(7)$ & $0.313(20)$ \\
\hline & $R$ & 3.4 & 18 & 4 & -2.50 & 0.07 & 0.89 & $24.65(8)$ & $0.417(30)$ \\
\hline & $I$ & 4.1 & 11 & 4 & -1.90 & 0.05 & 0.95 & $24.52(13)$ & $0.371(42)$ \\
\hline \multirow[t]{2}{*}{ Geminga } & $\bar{R}$ & 3.7 & 15 & 4 & -1.63 & 0.14 & 0.84 & $25.53(11)$ & $0.185(17)$ \\
\hline & $I$ & 3.9 & 10 & 4 & -1.37 & 0.05 & 0.87 & $25.10(14)$ & $0.217(26)$ \\
\hline
\end{tabular}

\section{Results}

The optical counterparts of both pulsars are firmly detected in all Subaru images with a signal to noise ratio $S / N \geq 10$. The instrumental magnitudes of the counterparts were measured for a range of circular aperture radii of $(1-3) \mathrm{CCD}$ pixels centered at the source positions. They were corrected for the point spread function (PSF) of bright stars. The background was measured over the annulus with the inner and outer radii $r_{\text {in }}=5$ and $r_{\text {out }}=10$ pixels. Within the measurement errors the results for different apertures coincided and a 2 pixel radius, or 4 pixels in the diameter that is comparable with the seeing of about 3-4 pixels (cf. Table 2), was adopted as an optimal to get a maximum $S / N$. Using Eq. (1) Johnson-Cousins magnitudes were derived and transformed into the fluxes in physical units using zero-points provided by Fukugita et al. (1995). The results are given in Table 4. The errors include statistical errors of the instrumental magnitude measurements, the errors of photometric referencing, and possible atmospheric extinction variations.

\subsection{Geminga}

\subsubsection{Identification in the $I$ band}

The object of $I \approx 25.1$ detected with the Subaru at the expected Geminga position is by a magnitude brighter than the upper limit $I \geq 26$. 4 reported by Mignani et al. (1998) based on previous $I$ band observations with the ESO/NTT. Such a deep limit is puzzling since it is comparable with the $3 \sigma$ detection limit of our Subaru observations, $I \geq 26$. 8 , obtained with similar exposure time ( $1.5 \mathrm{~h}$ vs. $1.4 \mathrm{~h}$ of NTT), but with a much higher telescope collective area ( $8 \mathrm{~m}$ vs. $3.6 \mathrm{~m}$ of NTT) and at better seeing conditions (0.' $6-0 .{ }^{\prime} 8$ vs. 0.' $9-1{ }^{\prime \prime} 11$ of NTT). Using the NTT/SUSI Exposure Time Calculator (ETC) ${ }^{8}$ one can easily estimate that at the respective conditions the NTT image should be by a magnitude less deep than the reported limit. This is in agreement with an upper limit of $I \geq 25.4$, which is presented in the text of the paper by Bignami et al. (1996) describing the NTT observations of Geminga. However, this reasonable limit was likely incorrectly reproduced in Figs. 3 and 4 of the same paper, and later on in Mignani et al. (1998).

To solve this puzzle we inspected the archival NTT image, which is shown in the left panel of Fig. 3. The expected position

\footnotetext{
8 eso.org/observing/etc/
}
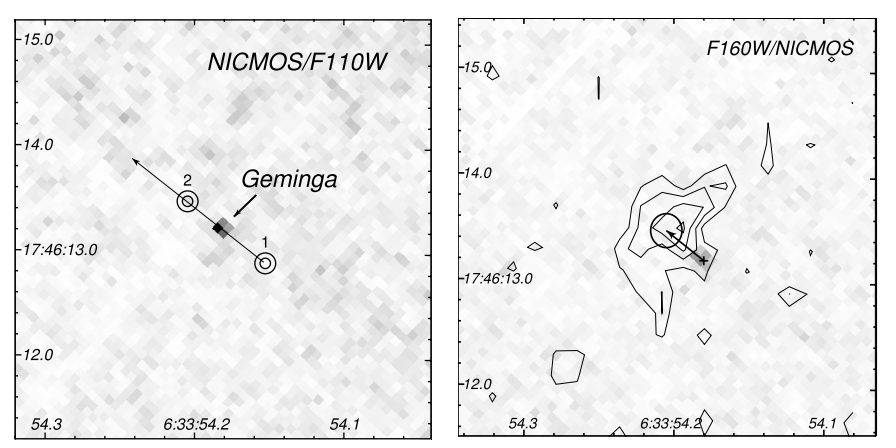

Fig. 4. $4^{\prime \prime} \times 4$ " images of Geminga in the F110W (left) and F160W (right) bands. The circle annulus in the left panel mark the expected Geminga positions at the epochs of I-band observations with the NTT (1) and Subaru (2), respectively, while the long arrow shows the pulsar path calculated based on the proper motion measurements of Caraveo et al. (1996). Contours of the Subaru image in $I$ band are overlaid in the right panel where the arrow shows the shift of Geminga due to its proper motion over $3.2 \mathrm{yr}$ between the HST and Subaru observations; "+" and $1 \sigma$-ellipse mark the expected positions of the pulsar at the epochs of the HST and Subaru observations, respectively.

of Geminga at the epoch of the NTT observations, 1995.084, is marked by a cross and the proper motion of the pulsar is shown by a dashed arrow. Several background-like objects are seen in this image in a few arcseconds from the position of Geminga, while the pulsar itself can be hardly identified in this image. A thorough comparison with the Subaru I-band image (bottom panel of Fig. 2), whose contours are overlaid, shows that these objects are likely to be artifacts caused by a poor data reduction in the absence of the standard flatfield image in the archive (cf. Sect. 2.4). This is further strengthened by the fact that there are no background objects in this region detected neither in our Subaru $R$ band (top panel of Fig. 2) nor in the HST F110W band images (Figs. 3, 4) which are adjacent to $I$ band in the spectral energy distribution and where Geminga is clearly identified. Our rough estimate of the $3 \sigma$ detection limit of the NTT image shown in Fig. 3 gives $I \geq 24$. 4 . This is by a magnitude less deep than the above ETC and those of Bignami et al. (1996) estimates and can be caused by the data reduction problems mentioned above. However, it is hardly possible to get a two magnitude deeper flux limit, as in Mignani et al. (1998), by any improvement of the NTT data reduction. Therefore, it has been overestimated at least by a magnitude for 
Table 5. Geminga in the NIR-optical-NUV-FUV range.

\begin{tabular}{|c|c|c|c|}
\hline $\begin{array}{l}\text { Filter/ } \\
\text { Telescope }\end{array}$ & $\log v$ & $\begin{array}{c}F_{v} \\
\text { observed } \\
\mu \mathrm{Jy}\end{array}$ & $\begin{array}{c}F_{v} \\
\text { dereddened }^{a} \\
\mu \mathrm{Jy}\end{array}$ \\
\hline \multicolumn{4}{|c|}{ NIR } \\
\hline F160W/HST & $14.273\left({ }_{-51}^{+58}\right)$ & $0.230(25)^{b, c}$ & $0.233(25)$ \\
\hline F110W/HST & $14.436\left(\left(_{-105}^{+138}\right)\right.$ & $0.166(21)^{b, c}$ & $0.170(21)$ \\
\hline \multicolumn{4}{|c|}{ Optical } \\
\hline I/Subaru & $14.571\left({ }_{-40}^{+44}\right)$ & $0.193_{-21}^{+19 c}$ & $0.201_{-22}^{+20}$ \\
\hline$R /$ Subaru & $14.658\left({ }_{-49}^{+55}\right)$ & $0.172_{-18}^{+17 c}$ & $0.181_{-19}^{+18}$ \\
\hline$F 555 W / \mathrm{HST}$ & $14.734\left({ }_{-54}^{+62}\right)$ & $0.170(20)^{d}$ & $0.182(21)$ \\
\hline$F 555 W / \mathrm{HST}$ & $14.734\left({ }_{-54}^{+62}\right)$ & $0.146(13)^{c}$ & $0.156(14)$ \\
\hline F430W/HST & $14.882\left({ }_{-44}^{+49}\right)$ & $0.146(11)^{c}$ & $0.160(12)$ \\
\hline \multicolumn{4}{|c|}{ NUV } \\
\hline$F 342 W / \mathrm{HST}$ & $14.944\left({ }_{-43}^{+47}\right)$ & $0.201(10)^{c}$ & $0.224(11)$ \\
\hline$F 25 S R F / \mathrm{HST}$ & $15.116\left({ }_{-95}^{+122}\right)$ & $0.261(26)^{d}$ & $0.315(31)$ \\
\hline$F 195 W / \mathrm{HST}$ & $15.104\left({ }_{-39}^{+159}\right)$ & $0.288(30)^{c}$ & $0.344(36)$ \\
\hline \multicolumn{4}{|c|}{ FUV } \\
\hline$G 140 L / \mathrm{HST}$ & $15.322(84)$ & $0.455(29)^{d}$ & $0.546(35)$ \\
\hline
\end{tabular}

${ }^{a} E(B-V)=0.023 ;{ }^{b}$ Harlow et al. (1998); ${ }^{c}$ this work; ${ }^{d}$ Kargaltsev et al. (2005) using ACS and STIS/MAMA NUV and FUV.

unknown reasons. On the other hand, we note that the object detected at the Geminga position in the Subaru $I$ image could be likely detected also in the NTT image with $S / N \sim 4$, provided a straightforward NTT data reduction is possible. This would be consistent with the significance of a marginal detection of Geminga in the $I$ band with the $6 \mathrm{~m}$ BTA telescope reported by Kurt et al. (2001).

To insure further our $I$ band and NIR identification of Geminga we estimated the shift between the counterpart positions in the HST/NICMOS and Subaru images (see Fig. 4). It was found to be $0{ }^{\prime} 45 \pm 0.22$. Despite the only $2 \sigma$ significance of this estimate it is in a good agreement with the shift direction (marked by arrows in Fig. 4) and with its value of $0.31 \pm 0$. 01 , which are expected from the Geminga proper motion (Caraveo et al. 1996; Table 1) at the time base of about $3.2 \mathrm{yr}$ separating the HST and Subaru observations. We have not found also any reliable faint red background source on the path of the pulsar proper motion, which would prevent from correct identification of Geminga in the $I$ band at epochs after the NTT observations, as was warned by Mignani (private communication). This allows us to make a conclusion on a firm identification of Geminga in the $I$, as well as in $R$, and in the two NIR bands.

\subsubsection{Broadband fluxes from the NIR through FUV}

The results of our I-R and NIR photometry of Geminga are presented in Table 5 where we have collected also most accurate and partially updated results of available pulsar observations in other broad bands. The flux in the $R$ band is in a good agreement with what was published by Mignani et al. (1998), but the accuracy of the Subaru observations is considerably higher. The result in the $I$ band confirms a tentative detection reported by Kurt et al. (2001), but at much higher significance level. The NIR fluxes are in agreement with the preliminary results published by Koptsevich et al. (2001) and Komarova et al. (2003).

Our reanalysis of the four HST/WFPC2 datasets obtained at different epochs in the $F 555 \mathrm{~W}$ band in the period of 1994-1995 yield the Geminga fluxes in the range of $0.136-0.161 \mu \mathrm{Jy}$ depending on the set. The flux values in separate sets are consistent with each other and do not show any variation of the Geminga emission with time above $3 \sigma$ level. In Table 5 we show a mean $F 555 W$ flux over these sets. It is also consistent with the value obtained recently with the HST/ACS in the same band (Kargaltsev et al. 2005; cf. Table 5) but a factor of 1.7 smaller than early results (Bignami et al. 1996; Mignani et al. 1998). This provides independent confirmations of a significant overestimation of the Geminga flux in the $F 555 \mathrm{~W}$ band in the previous publications, as it has been noticed by Kargaltsev et al. (2005). At the same time, our reanalysis of the HST/FOC archival data obtained in $F 430 \mathrm{~W}, F 342 \mathrm{~W}$, and $F 195 W$ bands shows a less significant, about $2 \sigma$ (or $10 \%-20 \%$ of the flux value), but systematic underestimation of the previously published fluxes. In Table 5 we present the updated flux values in these bands.

\subsubsection{Possible bow-shock structure}

Observations of the Geminga field in X-rays with the XMM have revealed a faint bow-shock structure around this pulsar whose tails are extended up to 2.5 behind the moving NS (Caraveo et al. 2003). Bow-shock structures produced by interaction of supersonically moving NSs with ambient medium have been detected around several pulsars presumably in the $\mathrm{H}_{\alpha}$ emission (e.g., the Guitar nebula around PSR B2224+65; Cordes et al. 1993), where they are usually easier detected than in X-rays (e.g., Romani et al. 1997). However, no signs of any $\mathrm{H}_{\alpha}$ counterpart of the Geminga shock structure were detected at deep imaging carried out in narrow $\mathrm{H}_{\alpha}$ bands with the VLT (Caraveo et al. 2003) and 6m BTA (Komarova et al. 2005). A faint $\mathrm{H}_{\alpha}$ counterpart, if exists, is likely hidden in a rather strong extended background emission in the $\mathrm{H}_{\alpha}$ line from a low Galactic latitude region where Geminga is located $(b \sim 4.7)$.

At the same time, as in case of the Crab pulsar nebula (Hester et al. 2002), the brightness of the shocked structures of Geminga could be higher in the optical continuum instead of lines. In this case they have to be more easier detected with broadband observations. A careful inspection of the Subaru images has allowed us to find possible indications of the optical counterpart of the X-ray structure in the deepest $I$ band image. A faint arc-like feature resembling a head of the bow-shock can be seen in Fig. 5. Its contours are shown in the right panel of Fig. 4. The arc length is $\sim 1^{\prime \prime} .9$ and it extends perpendicular to the pulsar path. There are no signs of this structure in our less deep $R$ band image as well as in the archival HST images. Further deep imaging of the field is needed to understand whether the arc-like structure is indeed a counterpart of 


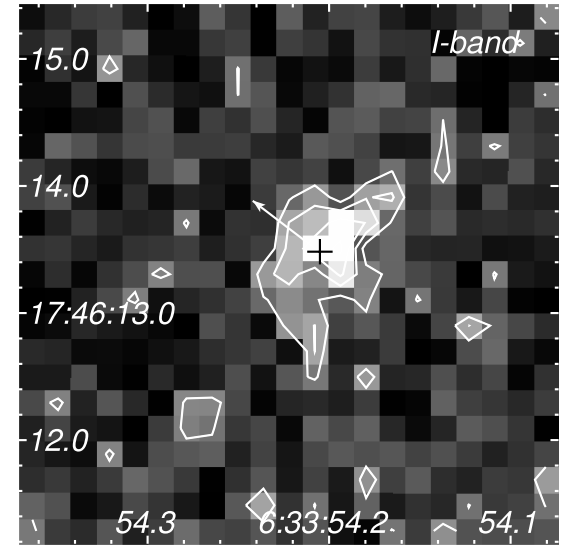

Fig. 5. A magnified fragment of the Subaru $I$ band image of Geminga demonstrating an arc-like structure resembling a head of a bow-shock due to the pulsar supersonic motion in the interstellar matter (cf. contours in Fig.4). The motion direction is shown by arrow, the pulsar position is marked by a cross.

the bow-shock seen in X-rays, a faint background extended object, or an artifact.

\subsection{PSR B0656+14}

\subsubsection{Broadband fluxes from the NIR through NUV}

Previous photometric measurements of the pulsar fluxes in the $B, R$, and, especially in the $I$ band (Kurt et al.1998; K2001) were rather uncertain due to poor seeing conditions and a contamination from a faint extended background object o 2 which sits only in 1 .' 1 from the pulsar (cf. Fig. 1). Perfect seeing during the Subaru observations allowed us to avoid the contamination and to measure for the first time the fluxes in these bands with the accuracy of better than $10 \%$. The results of our photometry of the pulsar are shown in Table 6, where the most accurate and informative data in other broad bands are also collected ${ }^{9}$.

\subsubsection{Possible PWN structure}

As in case of Geminga we find also a hint of a faint compact nebula around PSR B0656+14 possibly associated with the pulsar activity. This is seen in the deepest image obtained in the $I$ band (Fig. 6). The detected structure is elongated approximately perpendicularly to the direction of the pulsar proper motion. Its maximal extension is $\sim 1^{\prime \prime}$. 5 . If this is not a background object or an artifact, it is the second indication of the presence of a faint compact PWN or a bow shock nebula around PSR B0656+14 in the optical range. The first one was mentioned by Koptsevich et al. (2001) who found a knot-like structure seen at a $3 \sigma$ level above the wings of the pulsar spatial profile at $\sim 0$ ' $^{\prime} 04$ from the pulsar position in the archival HST/FOC/F343W image. Knot-like structures are typical

\footnotetext{
${ }^{9}$ As in case of Geminga (Sect. 3.1), using archival data we have examined the published fluxes of PSR B0656+14 obtained with different HST instruments. No significant discrepancies from the results as they are listed in K2001 were found.
}

Table 6. PSR B0656+14 in the NIR-optical-NUV range.

\begin{tabular}{|c|c|c|c|}
\hline $\begin{array}{l}\text { Filter/ } \\
\text { Telescope }\end{array}$ & $\log v$ & $\begin{array}{c}F_{v} \\
\text { observed } \\
\mu \mathrm{Jy}\end{array}$ & $\begin{array}{c}F_{v} \\
\text { dereddened }^{a} \\
\mu \mathrm{Jy}\end{array}$ \\
\hline \multicolumn{4}{|c|}{ NIR } \\
\hline$F 187 W / \mathrm{HST}$ & $14.204\left({ }_{-28}^{+30}\right)$ & $0.779(97)^{b}$ & $0.789(98)$ \\
\hline F160W/HST & $14.273\left({ }_{-51}^{+58}\right)$ & $0.575(41)^{b}$ & $0.584(42)$ \\
\hline F110W/HST & $14.436\left(\begin{array}{c}+138 \\
+105\end{array}\right)$ & $0.336(30)^{b}$ & $0.346(31)$ \\
\hline \multicolumn{4}{|c|}{ Optical } \\
\hline I/Subaru & $14.571\left({ }_{-40}^{+44}\right)$ & $0.371\left(\left(_{-40}^{+44}\right)^{c}\right.$ & $0.390\left(\left(_{-42}^{+46}\right)\right.$ \\
\hline$R /$ Subaru & $14.658\left({ }_{-49}^{+55}\right)$ & $0.417\left({ }_{-49}^{+55}\right)^{c}$ & $0.447\left({ }_{-53}^{+59}\right)$ \\
\hline F555W/HST & $14.734\left({ }_{-54}^{+62}\right)$ & $0.393(24)^{b}$ & $0.428(26)$ \\
\hline$B /$ Subaru & $14.829\left({ }_{-47}^{+52}\right)$ & $0.313\left({ }_{-47}^{+52}\right)^{c}$ & $0.350\left({ }_{-53}^{+58}\right)$ \\
\hline F430W/HST & $14.882\left({ }_{-44}^{+49}\right)$ & $0.264(29)^{d}$ & $0.300(33)$ \\
\hline \multicolumn{4}{|c|}{ NUV } \\
\hline$F 342 W / \mathrm{HST}$ & $14.944\left({ }_{-43}^{+47}\right)$ & $0.307(27)^{d}$ & $0.353(31)$ \\
\hline GRISM/HST & $15.071\left({ }_{-125}^{+170}\right)$ & $0.354(31)^{e}$ & $0.429(38)$ \\
\hline F195W/HST & $15.104\left(\begin{array}{l}+39 \\
-159\end{array}\right)$ & $0.349(43)^{d}$ & $0.438(54)$ \\
\hline
\end{tabular}

${ }^{a} E(B-V)=0.03 ;{ }^{b} \mathrm{~K}(2001) ;{ }^{c}$ this work; ${ }^{d}$ Pavlov et al. (1997); ${ }^{e}$ Shibanov et al. (2005).

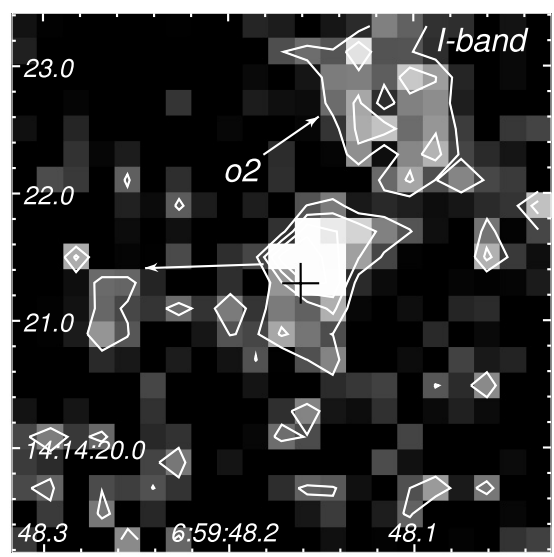

Fig. 6. Magnified fragment of the Subaru $I$ band image of PSR B0656+14 demonstrating possible existence of a PWN or a bowshock structure around the pulsar (extended object in the center of the image). Contours are overlaid to underline the shape of the structure; the arrow shows the direction of the pulsar proper motion, the pulsar position is marked by a cross

attributes of the Crab PWN. However, in case of PSR B0656+14 both indications still have to be considered with caution and need to be confirmed by deeper observations since we found no signs of the nebula in any other available optical images of the pulsar. However, all these images are less deep than the Subaru $I$ band image.

\section{Discussion}

Reliable detection of Geminga and PSR B0656+14 in the $I$ and $R$ bands basically completes a comprehensive broadband photometric study of both pulsars in the NIR through FUV range started about a dozen years ago. As a result, the two pulsars have been detected at a high significance level 
practically in all standard optical bands generally used for the photometry of ordinary stars to obtain the spectral energy distributions of their radiation in a wide spectral domain. Except for the most bright and younger Crab and Vela pulsars, Geminga and PSR B0656+14 stand up to be the most studied up to date radio pulsars in the optical range.

\subsection{Spectral energy distribution in the optical range 4.1.1. Extinction corrections}

Interstellar color excesses $E(B-V)$ along the lines of sight to Geminga and PSR B0656+14 have not yet been investigated accurately. This requires additional careful optical studies of suitable stars from the pulsar neighborhoods. So far there are only indirect estimations of the extinction based on observations in other spectral domains (e.g., Pavlov et al. 1996; Kurt et al. 1998; Kurt et al. 2001). Closeness of both pulsars to the Galaxy plane (see Table 1) suggests that their optical fluxes are to be affected by the foreground extinction from the Milky Way. On the other hand, the reddening cannot be too strong due to their proximity to the Earth. This is confirmed by relatively low DMs and hydrogen column densities $N_{\mathrm{H}}$ obtained from the radio and $\mathrm{X}$-ray observations. The conservative $N_{\mathrm{H}}$ values are $\approx 1.1 \times 10^{20} \mathrm{~cm}^{-2}$ for Geminga based on the ROSAT and ASCA X-ray spectral observations (Halpern \& Wang 1997; Jackson et al. 2002) and $\approx 1.4 \times 10^{20} \mathrm{~cm}^{-2}$ for PSR B0656+14 based on the ROSAT, ASCA and EUVE observations (K2001). Using these values and an empirical relation $N_{\mathrm{H}} / E(B-V)=4.8 \times 10^{21} \mathrm{~cm}^{-2} \mathrm{mag}^{-1}$ applicable for the Milky Way (Bohlin et al. 1978) one can obtain $E(B-V)$ of 0.023 and 0.03 for Geminga and PSR B0656+14, respectively. These values are consistent with those suggested by previous studies. Apparently smaller value of $E(B-V)$ for Geminga is compatible with the fact that it is by about $100 \mathrm{pc}$ closer to us than PSR B0656+14. We applied these excesses to calculate dereddened fluxes in a standard way making use of mean Galactic extinction curve (Cardelli et al. 1989) and the de-reddening parameter $R(V)=3.1$. The results are presented in Tables 5 and 6 . For both pulsars the dereddening corrections are within $1 \sigma$ of the flux uncertainties in the optical-NIR range, while they are significant in the UV.

We note that spectral analysis of recent Chandra and XMM $\mathrm{X}$-ray observations with previously used standard spectral models give systematically higher $N_{\mathrm{H}}$ values than those of the ASCA and ROSAT: $\approx 2.4 \times 10^{20} \mathrm{~cm}^{-2}$ for Geminga (Kargaltsev et al. 2005) and $\approx 4.3 \times 10^{20} \mathrm{~cm}^{-2}$ for PSR B0656+14 (De Luca et al. 2005). The latter one corresponds to $E(B-V) \approx 0.09$ which is comparable with the extinction throughout the entire Galaxy in this direction (Schlegel et al. 1998) and hardly can be relevant for the nearby pulsar. $N_{\mathrm{H}}$ for Geminga appears to be also overestimated and we skip out these implausibly high values ${ }^{10}$ from our consideration and use the above ones provided

\footnotetext{
10 The overestimation of $N_{\mathrm{H}}$ in both cases is likely due to the poorly calibrated instrument responses in the energy range of $\lesssim 0.3 \mathrm{keV}$ (e.g., Kargaltsev et al. 2005) which is most crucial for $N_{\mathrm{H}}$ estimates. This also leads to lower temperatures of thermal emission components and to higher spectral indices of nonthermal components derived from spectral fits.
}

by early ASCA, EUVE, and ROSAT data analyses. We note, that $N_{\mathrm{H}}$ was also fixed at the ROSAT value in the recent analysis of the Geminga X-ray spectrum based on the most complete data set taken with the XMM (Caraveo et al. 2004).

\subsubsection{Broadband spectra from NIR through UV}

The dereddened broadband spectral energy distributions of the two pulsars are shown in Fig. 7. Despite a factor of 2-3 difference between the mean flux levels of the two objects and higher flux uncertainties in case of Geminga, the spectral shapes of the optical emission of both middle-aged pulsars are remarkably similar to each other. Both are clearly nonmonotonic with apparent dips in the $F 430 \mathrm{~W}$ and $F 110 \mathrm{~W}$ bands and excesses centered in the $V-I$ range. PSR B0656+14 has also a significant flux increase towards lower frequencies in the NIR range. Similar increase is seen in the spectrum of Geminga but it is less significant due to the absence of observations in the $K$ band (or in its HST analog $F 187 W$ ). There is also flux increase towards higher frequencies in the UV range which is most prominent for Geminga where the FUV data are available. The spectroscopic data recently obtained for PSR B0656+14 in the NUV (Shibanov et al. 2005) and for Geminga in the FUV (Kargaltsev et al. 2005) are also shown in Fig. 7 for comparison. They are compatible with the broadband data, but the flux uncertainties (not shown) in spectral bins are considerably larger than photometric ones, and apparent absorption/emission features in the spectra have a low significance of $\lesssim 2 \sigma$.

Resulting spectral energy distributions cannot be fitted by a single power-law, as it is possible for the young Crab pulsar in the whole observed range (Sollerman 2003; Sollerman et al. 2000). This suggests a multicomponent structure of the emission of the two middle-aged pulsars and may reveal spectral evolution of the optical emission with pulsar age (K2001; Shibanov et al. 2003; Zharikov et al. 2004). Some of the components can be identified from the comparison with the X-ray data.

\subsubsection{Thermal and nonthermal spectral components}

For PSR B0656+14 the flux increase with frequency in the UV can be explained by a contribution of the Rayleigh-Jeans part of the thermal blackbody-like spectral component (BB) from the whole surface of the NS strongly dominating the pulsar emission in soft X-rays. The flux increase towards the infrared appears to be consistent with the low energy extension of the power law component (PL) seen in the high energy tail of the X-ray spectrum. The unabsorbed PL and BB components and their sum are shown by dashed lines in Fig. 7. Their parameters are taken from K2001: the BB temperature $T_{\mathrm{BB}} \approx 0.84 \mathrm{MK}$ and the radius of the emitting area $R_{\mathrm{BB}} \approx 7.8 \mathrm{~km}$ at the distance $d \approx 288 \mathrm{pc}$; the PL spectral index $\alpha_{v} \approx-0.45$ and normalizing constant at $1 \mathrm{keV}$ is $\approx 2.8 \times 10^{-5} \mathrm{ph} \mathrm{cm}^{-2} \mathrm{~s}^{-1} \mathrm{keV}^{-1}$; $N_{\mathrm{H}} \approx 1.42 \times 10^{20} \mathrm{~cm}^{-2}$. The $N_{\mathrm{H}}$ value and $E(B-V)$ in the plot are consistent with each other as discussed above. Uncertainties of the X-ray spectral fit extension are shown by a dashed 

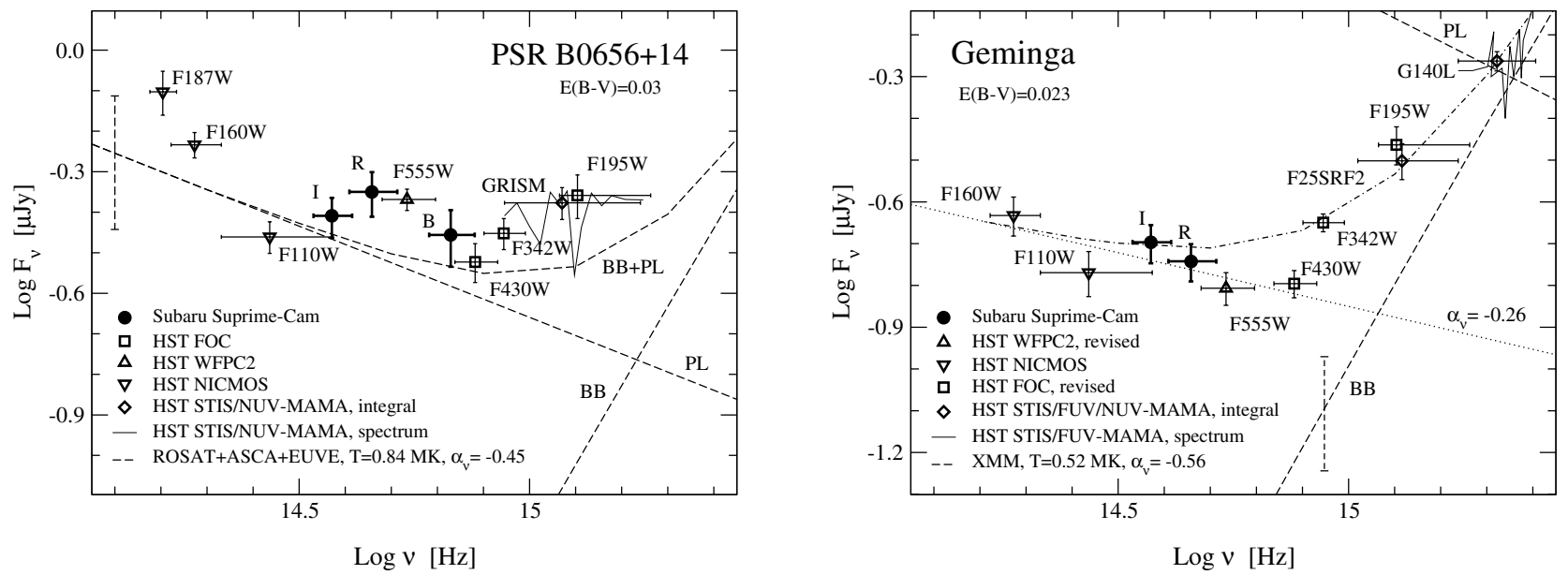

Fig. 7. Dereddened NIR-UV broadband spectra of PSR B0656+14 (left) and Geminga (right) summarizing the best up to date quality observations obtained with different telescopes, instruments and filters, as indicated in the plots and listed in Tables 5 and 6 . Note a nonmonotonic structure and remarkable similarity of the broadband spectra for both pulsars. Thin solid curves show the spectroscopic data obtained for PSR B0656+14 in the NUV (Shibanov et al. 2005) and for Geminga in the FUV (Kargaltsev et al. 2005). Dashed lines are the low energy extensions of the unabsorbed soft blackbody (BB) and power law (PL) spectral components derived from the combined BB $+\mathrm{BB}+\mathrm{PL}$ spectral fits in X-rays. Parameters of the fits are taken from Koptsevich et al. (2001) for PSR B0656+14 and from Kargaltsev et al. (2005) for Geminga. The second, hard BB components are negligible for both pulsars in the NIR-UV range and not shown in the plots. Dotted and dot-dashed lines in the right panel show the PL fit of the Geminga optical data in the range from F160W through F430W bands and its sum with the BB, respectively (see Sect. 4.1.3 for details).

errorbar. As seen from the left panel of Fig. 7, the BB+PL extension qualitatively fits the spectrum of PSR B0656+14, albeit the PL component dominates in the most part of the observed optical range. This is also confirmed by detailed analyses of the pulsar pulse profile and the spectrum in the NUV, where the BB contribution was estimated to be $\$ 30 \%$ (Shibanov et al. 2005).

For Geminga the situation is more complex. The parameters of the X-ray extensions in this case are taken from Kargaltsev et al. (2005): $T_{\mathrm{BB}} \approx 0.52 \mathrm{MK}, R_{\mathrm{BB}} \approx 9.5 \mathrm{~km}$ at $d \approx 200 \mathrm{pc}$; the PL index $\alpha_{v} \approx-0.56$ and normalizing constant at $1 \mathrm{keV}$ is $\approx 5.5 \times 10^{-5} \mathrm{ph} \mathrm{cm}^{-2} \mathrm{~s}^{-1} \mathrm{keV}^{-1} ; N_{\mathrm{H}}$ was fixed during the X-ray spectral fit at the value of $1.1 \times 10^{20} \mathrm{~cm}^{-2}$. As seen from the right panel of Fig. 7, the PL and, therefore, the sum $\mathrm{BB}+\mathrm{PL}$ (it is outside the upper plot frame) overshoot the optical fluxes of Geminga by a factor of $\gtrsim 100$. This fact has been noticed by Halpern \& Wang (1997) and confirmed later on by Jackson et al. (2002), Komarova et al. (2003), and Kargaltsev et al. (2005). At the same time, the BB extension appears to fit qualitatively well a steep flux increase of Geminga towards higher frequence range.

Various possibilities to resolve the discrepance between the low energy extension of the Geminga X-ray spectrum and the observed optical fluxes have been discussed by Kargaltsev et al. (2005), including possible systematic errors due to instrument response uncertainties, large statistical uncertainties of the X-ray PL component, its contamination by the hard BB component from the pulsar polar caps, and possible existence of a spectral break in the spectrum of the nonthermal component somewhere between the optical and X-ray ranges.

The similarity of the optical spectral shape of Geminga with that of PSR B0656+14 suggests that its low frequence tail is of nonthermal origin and can be fitted by a power law. Such a fit performed for the $F 160 \mathrm{~W}-F 430 \mathrm{~W}$ range is shown by a dotted line in the right panel of Fig. 7. Its sum with the BB extension (dot-dashed line) qualitatively fits the whole set of the data in the NIR-UV range, as it is in case of the $\mathrm{BB}+\mathrm{PL}$ extension for PSR B0656+14. The slope of the optical PL, $\alpha_{v} \approx-0.26$, is almost twice smaller than that in X-rays, $\alpha_{v} \approx-0.56$, favoring the interpretation with the spectral break in the nonthermal optical-X-ray emission of Geminga. Changing the spectral slope of the nonthermal component from almost a flat in the optical to a significantly negative in X-rays is not unusual and has been observed in the emission of the young Crab pulsar (Kuiper et al. 2001; Sollerman 2003), older Vela (Shibanov et al. 2003; Romani et al. 2005), and likely in two old pulsars PSR B1929+10 and PSR B0950+08 detected in the optical (Pavlov et al. 1996; Mignani et al. 2002; Zharikov et al. 2002, 2004) and X-rays (Becker et al. 2004, 2005; Zavlin \& Pavlov 2004b). The nonthermal emission of very old isolated millisecond pulsars studied in the optical and X-ray ranges shows even a much stronger slope break between these ranges (Koptsevich et al. 2003; Mignani \& Becker 2004). We discuss the possible position of the break for Geminga below in Sect. 4.2.

\subsubsection{Third optical spectral component?}

A wide $F 555 W-R$ excess in the emission of PSR B0656+14 cannot be explained by only the combination of the RayleighJeans and power-law components. It exceeds by $\sim(3-5) \sigma$ a power-law "continuum level" supposed to be seen in the $F 430 W$ and $I$ bands. The excess may reveal a new, not yet identified, third spectral component in the emission of PSR B0656+14. 
The nature of the excess is unclear. One can speculate that it is produced by unresolved emission lines from a compact PWN around the pulsar interacting with interstellar environment polluted by heavy elements from the $\mathrm{SN}$ explosion associated with the pulsar. Strong OIII (5007 ̊), SII (6717 ̊) and other fainter nebular lines, whose wavelengths are within the spectral range of the excess, are typical for the spectra of bright and extended PWNe formed around young pulsars like Crab. In case of PSR B0540-69 a strong background emission in OIII (5007 $\AA$ ) line from the SNR core contaminates the pulsar photometric flux in the $F 555 \mathrm{~W}$ band and results in a false flux excess exceeding by an order of a magnitude the real flux from the pulsar (Serafimovich et al. 2004). A distant outer shell of the PSR B0656+14 SNR has been detceted in X-rays (Nousek et al. 1981; Thorsett et al. 2003). However, nothing is known on the presence of the nebular lines in the pulsar vicinity, although they could exist if the pulsar PWN marginally detected in the optical (Fig. 6) and X-rays (Marshall \& Schulz 2002) is real.

Alternatively, the excess can be an internal property of the pulsar emission. It can be produced by ion or electron cyclotron features formed in magnetospheric plasma of a strongly magnetized NS. The features are expected to be broad enough due to the magnetic field inhomogeneity at the magnetospheric spatial scale. Current data do not allow us to discriminate between the two possibilities. To do that a deep narrow-band imaging of the pulsar field with a standard set of narrow-band filters centered at nebular lines and/or spectroscopy of the pulsar are necessary.

Geminga is fainter and its fluxes are determined with larger errors. The apparent I-R excess in its spectrum is much less significant than for PSR B0656+14. However, the similarity of the spectral shapes of both pulsars provides an additional argument that this excess is real. This is also supported by the presence of the X-ray nebulosity around Geminga and its possible optical counterpart (Fig. 5), which may be resposible for the excess. In this case, one can suggest the same two possibilities to explain its nature as has been discussed above for PSR B0656+14.

We note, that with the new optical and NIR data the optical spectrum of Geminga cannot be described by a two component model (Jacchia et al. 1999) combining only an ion cyclotron emission line from a hot plasma near the polar caps of the pulsar and the thermal radiation from the cooling surface of the NS. The nonthermal PL spectral component has to be included into the interpretation and it strongly dominates the emission at longer wavelengths.

\subsection{Multiwavelength spectra}

In Fig. 8 we show unabsorbed rotation-phase integrated multiwavelength spectra of both pulsars using available data from the radio through gamma-rays. The radio data for Geminga are kindly provided by Malofeev (private communication). There are some doubts on the radio detection (Burderi et al. 1999) and the data are represented mainly by upper limits. For PSR B0656+14 the radio data are from K2001. The optical data are from Tables 5 and 6 . The parameters of the X-ray spectral components are the same as in Fig. 7. For both NSs we add here also the hard BB components believed to be from the pulsar polar caps. They contribute significantly to the resulting spectra only near $\sim 1 \mathrm{keV}$ where the soft BB components meet the PL tails. Parameters of the hard BBs are from $\mathrm{K} 2001$ for PSR B0656+14 $(R \approx 0.52 \mathrm{~km}$ at $d \approx 288 \mathrm{pc}$, $T \approx 1.65 \mathrm{MK}$ ) and from Kargaltsev et al. (2005) for Geminga $(R \approx 0.046 \mathrm{~km}$ at $d \approx 200 \mathrm{pc}, T \approx 2.32 \mathrm{MK}$ ). All X-ray components are extended to the optical. The gamma-ray data are from Ramanamurthy et al. (1996) for PSR B0656+14 and from Jackson et al. (2002, RXTE), Macomb \& Gehrels (1999, OSSE, COPMTEL, EGRET) ${ }^{11}$, Akerlof et al. (1993, Whipple) and Vishwanath et al. (1993, Tata group) for Geminga.

The multiwavelength spectra underline the fact that a smooth connection of the optical and X-ray nonthermal and thermal spectral components observed for PSR B0656+14 is problematic for Geminga. Geminga is obviously underluminous in the optical range as compared with PSR B0656+14 and with the low-energy extension of its power-law component from X-rays. This suggests changing the spectral slope of the nonthermal component of Geminga from a significantly negative in X-rays ( $\alpha_{v} \approx-0.56$, dashed line) to almost a flat $\left(\alpha_{v} \approx-0.26\right.$, dotted line) in the NIR-optical range. A simple extrapolation of the NIR-optical PL fit to X-rays shows that the spectral break in the nonthermal optical-X-ray emission may occur near $1 \mathrm{keV}$ where the hard BB component dominates the total flux (bottom panel of Fig. 8).

A common origin of the optical and X-ray nonthermal components independently on pulsar age and the presence of spectral breaks was proposed by Zharikov et al. $(2004,2005)$ based on a strong correlation of the optical and X-ray nonthermal luminosities for a set of ordinary pulsars detected in the optical and X-ray domains (see also Zavlin \& Pavlov 2004b). Analysis of the phase aligned optical and X-ray pulse profiles would be useful to verify this hypothesis for Geminga, as it has been partly done for PSR B0656+14 (Shibanov et al. 2005). Detection of the optical pulsations of Geminga at a higher $S / N$ than it is provided by a tentative detection by Shearer et al. (1998) is necessary for this goal.

The multiwavelength nonthermal spectrum of Geminga suggests also at least three additional spectral breaks in the high energy range: in hard X-rays, in gamma-rays, and in the $\mathrm{TeV}$ range. They form a "knee-like" spectral structure of the high energy emission. Similar "knee" is seen in the high energy spectrum of the Vela-pulsar (e.g., Shibanov et al. 2003; Romani et al. 2005, and references therein). The optical part of the Vela spectrum suggests also a "degenerate knee" between the optical and X-rays while its NIR and X-ray data from the nonthermal spectral tail can be fitted by a single power-law (Shibanov et al. 2003). It is not clear whether the optical-X-ray and X-ray-gamma-ray knees are correlated or physically related to each other. The Crab pulsar does not have such a strong spectral breaks and knees in the high energy range, while it has a strong spectral break between the optical and X-rays (Kuiper et al. 2001; Sollerman 2003). The same appears to be true for PSR B0540-69 (Serafimovich et al. 2004). Various slopes and

11 cossc.gsfc.nasa.gov/docs/cgro/gamcat/ 

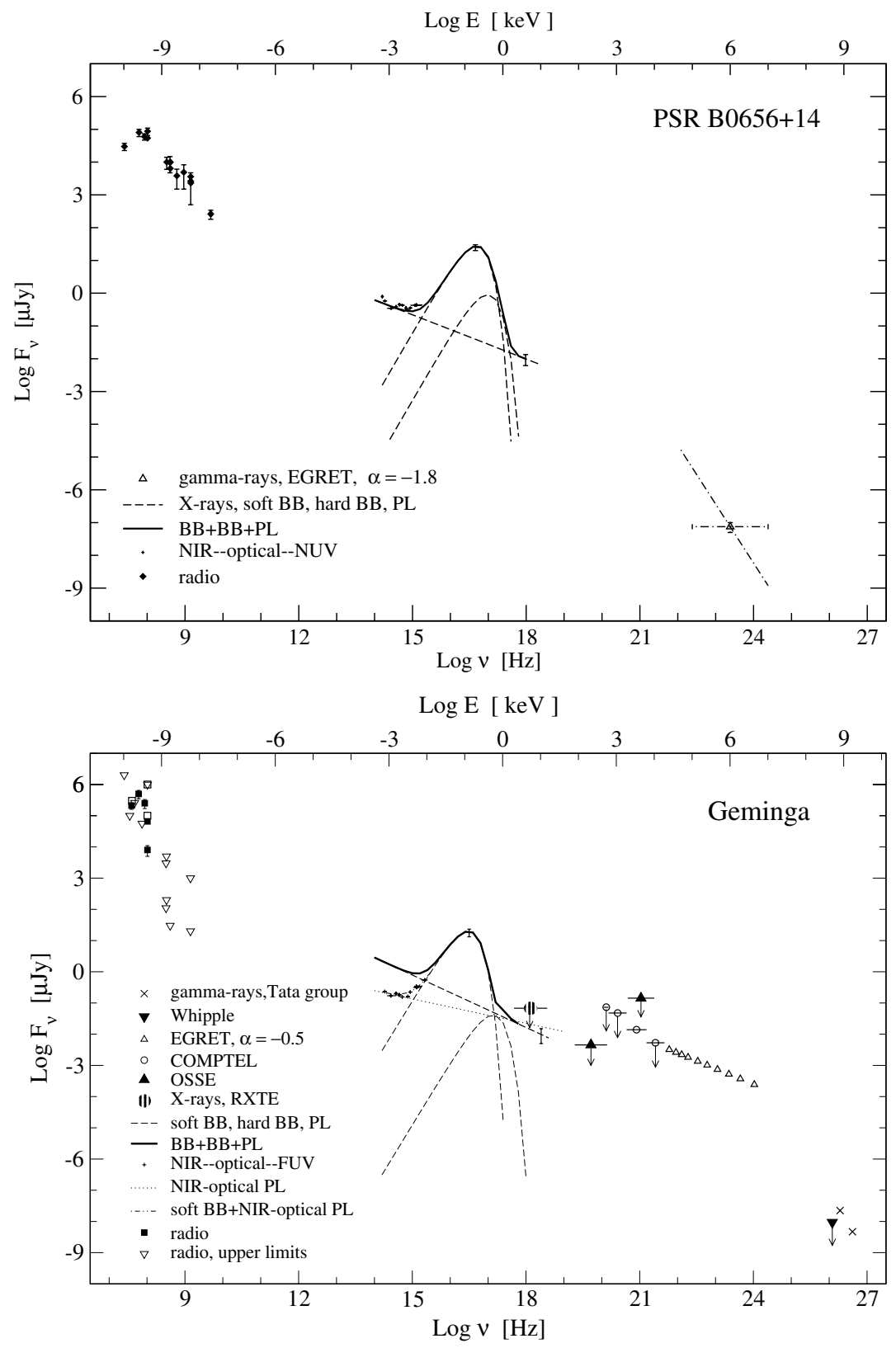

Fig. 8. Unabsorbed multiwavelength spectra of the two pulsars using available data from radio through gamma-rays, as notified in the plot. X-ray spectral components are extended to the optical range, while the optical one - to X-rays. numerous breaks likely suggest a multicomponent structure of the emission generated by different mechanisms or by different populations of the relativistic particle, or both. However, multiwavelength data for pulsars are too scarce to make any definite conclusion. For instance, PSR B0656+14 appears to have no significant spectral break between the optical and X-rays, while almost nothing is known on its high energy spectrum except it is likely softer than that of Geminga.

The problem of the spectral difference of the pulsars outside the radio range is far from its final solution. A question, whether this difference simply caused by different pulsar emission beaming and geometry factors or by significantly different physical conditions in the pulsar magnetospheres, is not answered yet. Studying the pulse profiles in different spectral ranges and phase resolved spectroscopy can help to solve this problem and, particularly, to understand whether the optical and X-ray nonthermal photons are generated at the same site and by the same population of relativistic particles accelerated in the pulsar magnetospheres. If the latter is true, then one has to understand why the distribution function of the particles in some cases reveals a break at an intermediate energy, which is reflected in the observed spectra.

\subsection{Possible optical PWNe}

The sizes of axisymmetrical equatorial torus-like (plus polar jets) PWNe around the young Crab, PSR B0540-69, and Vela pulsars are roughly scaled as $\dot{E}^{0.5}$, where $\dot{E}$ is the spin-down luminosity of a pulsar (Helfand et al. 2001; Pavlov et al. 2001; Serafimovich et al. 2004; Weisskopf et al. 2004). This is in agreement with the simplest model of a termination wind shock forming around subsonic-ally moving energetic pulsars due to the interaction of their relativistic winds with the ambient matter (Kennel \& Coroniti 1984). The PWNe of the Crab and its 
twin PSR B0540-69 are extended approximately to about $1 \mathrm{pc}$ from the pulsars and both have similar sizes in the optical and X-rays (e.g., Serafimovich et al. 2004). Using this value and the above scaling law one can estimate that PWNe around the middle-aged Geminga and PSR B0656+14 have to be about a factor of 100 smaller than in the Crab case, i.e., a few arcseconds at the distances to the pulsars of about 200-300 pc, provided the external density and pressure conditions are not much different from that of Crab and are appropriate to form the subsonic torus but not a supersonic bow shock. The sizes of the faint extended structures around both middle-aged pulsars marginally detected in our deepest Subaru images are in a reasonable agreement with these expectations. This is a starting point to discuss these structures as possible candidates for PWNe.

So far PWNe of a different nature and shapes have been detected in the radio, optical and X-rays around a dozen of isolated NSs (e.g., Gaensler 2005). Young NSs typically show the torus/jet-like PWNe, while the PWNe around old NSs commonly have a bow shock structure. Changing of the shock type with age is supposed to be due to a transformation of external conditions from the subsonic to supersonic ones when the density and the pressure within the expanding host SNR decrease to values of a typical ISM. A characteristic age of a host SNR when it dissolves in the ISM background roughly coincides with a middle-age epoch of the NS evolution, i.e., $10^{5}-10^{6} \mathrm{yr}$.

Following this scenario one can expect to see an intermediate PWN morphology, i.e., a mixture of torus and bowshock, around middle-aged pulsars. However, until now there are only two middle-aged pulsars with a faint, few arcseconds PWNe apparently detected only in the radio around PSR B0906-49 (Gaensler et al. 1998) and only in X-rays around PSR J0538+2817 (Romani \& Ng 2003). Both cases need additional observations in different wave bands to confirm the nature of the extended objects around the pulsars and to study their morphology. In this respect, adding to this poor sample any detection of PWNe, even a tentative, would be valuable.

In contrast to a bright Crab PWN, whose contribution to the total pulsar+nebula luminosity is about $95.5 \%$ and $99.8 \%$ in $\mathrm{X}$-rays and in the optical, respectively, the PWN-like structures around our middle-aged pulsars are faint and may contribute only a few percents to the total emission budget. However, this is compatible with the observational fact that the torus PWNe loose their brightness with age much sooner than the pulsars. For instance, the PWN contribution for an intermediate age Vela-pulsar $\left(10^{4} \mathrm{yr}\right)$ in X-rays is about $75 \%$ (Pavlov et al. 2001). It is likely to be much smaller in the optical range where the Vela-PWN has not yet been clearly identified (Shibanov et al. 2003; Mignani et al. 2003). A faint $\mathrm{X}$-ray PWN tentatively detected around a middle-aged pulsar PSR J0538+2817 $\left(6 \times 10^{5}\right.$ yr $)$ provides only about $2 \%$ of the pulsar flux in X-rays (Romani \& Ng 2003). This is in agreement with what is seen in our case of Geminga and PSR B0656+14.

Another factors suggesting that the extended optical structures around our pulsars are likely to be PWNe are their morphology and orientation to the vectors of the pulsar proper motion. The planes of the equatorial torus structures of young pulsars are typically arbitrary inclined to the line of sight and seen as elliptical extended objects. For the Crab, Vela, and, possibly, PSR B0540-69 pulsars the torus symmetry (pulsar spin) axis and PWN polar jets are aligned with the vector of proper motion of the pulsar, which may constrain the nature of the kick at pulsar birth (Serafimovich et al. 2004). Both properties are likely seen for Geminga and PSR B0656+14 where the extended optical structures (possible inclined tori) are aligned perpendicular to the pulsar proper motion, although any jet/counter-jet structures are not visible in our images.

Finally, possible pulsar nebulae around Geminga and PSR B0656+14 have been detected in X-rays, albeit at different significance levels. The sizes of a less significant circle X-ray nebulosity around PSR B0656+14 (Marshall \& Schulz 2002) are consistent with what is seen in the Subaru image. Geminga shows apparently more significant long (up to $\sim 2^{\prime}$ ) X-ray tails well aligned with the source proper motion (Caraveo et al. 2003). The tails evidence that the PWN of the older Geminga has transformed from the subsonic torus to the supersonic bow shock stage. This is also supported by a twice higher transverse velocity of Geminga, by absence of its host SNR, and, hence, likely smaller density and external pressure of the ambient matter than in case of PSR B0656+14. The latter one is younger and still sits inside its host SNR (Thorsett et al. 2003). Note also, that its DM is significantly and $N_{\mathrm{H}}$ is apparently higher than in the Geminga case, favoring a denser environment for PSR B0656+14. Consequently, an arc-like shape of the faint extended structure at the Geminga optical image suggests that it is likely to be a counterpart of the head of the bow-shock (but not a bright part of a PWN torus) whose tails are detected in X-rays.

Thus, the morphology of both optical nebulosities tentatively detected around the two middle-aged pulsars are roughly compatible with what is expected from their parameters and parameters of the environments, as well as with what is seen for each pulsar in X-rays. Confirmation of their reality at higher significance level and with better spatial resolution in the optical and X-rays would be valuable for understanding the physics of PWNe and their evolution with age.

Acknowledgements. We are grateful to Yutaka Komiyama for the help during observations with the Subaru, to Valeri Malofeev for radio data for Geminga, and to Roberto Mignani for the ESO/NTT optical data and stimulating comments. This work was supported in part by CONACYT project 25454-E, RFBR (grants 03-02-17423, 03-0790200 and 05-02-16245) and RLSS programma 1115.2003.2. Some of the data presented here were obtained from the Multi mission Archive at the Space Telescope Science Institute (MAST). STScI is operated by the Association of Universities for Research in Astronomy, Inc., under NASA contract NAS5-26555. Support for MAST for non-HST data is provided by the NASA Office of Space Science via grant NAG5-7584 and by other grants and contracts.

\section{References}

Akerlof, C. W., Breslin, A. C., Cawley, M. F., et al. 1993, A\&A, 274, L17 
Becker, W., \& Trümper, J. 1997, A\&A, 326, 682

Becker, W., Weisskopf, M. C., Tennant, A. F., et al. 2004, ApJ, 615, 908

Becker, W., Jessner, A., Kramer, M., Testa, V., \& Howaldt, C. 2005, ApJ, accepted [arXiv:astro-ph/0505488]

Bignami, G. F., \& Caraveo, P. A. 1996, ARA\&A, 34, 331

Bignami, G. F., Caraveo, P. A., Mignani, R., Edelstein, J., \& Bowyer, S. 1996, ApJ, 456, L111

Bohlin, R. C., Savage, B. D., \& Drake, J. F. 1978, ApJ, 224, 132

Brisken, W. F., Thorsett, S. E., Golden, A., \& Goss, W. M. 2003, ApJ, 593, L89

Burderi, L., Fauci, F., \& Boriakoff, V. 1999, ApJ, 512, L59

Caraveo, P. A., Bignami, G. F., Mignani, R., \& Taff, L. G. 1996, ApJ, 461, L91

Caraveo, P. A., Lattanzi, M. G., Massone, G., et al. 1998, A\&A, 329, L1

Caraveo, P. A., Bignami, G. F., De Luca, A., et al. 2003, Science, 301, 134

Caraveo, P. A., De Luca, A., Mereghetti, S., \& Bignami, G. F. 2004, Science, 305, 376

Cardelli, J. A., Clayton, G. C., \& Mathis, J. S. 1989, ApJ, 345, 245

Cordes, J. M., Romani, R. W., \& Lundgren, S. C. 1993, Nature, 362, 133

De Luca, A., Caraveo, P. A., Mereghetti, S., Negroni, M., \& Bignami, G. F. 2005, ApJ, 623, 1051

Fruchter, A. S., Hook, R. N., Busko, I. S., \& Mutchler, M. 1998, in 1997 HST Calibration Workshop, ed. S. Csertano, R. Jedrzejewsky, T. Keyes, \& M. Stevens (STScI)

Fukugita, M., Shimasaku, K., \& Ichikawa, T. 1995, PASP, 107, 945

Halpern, J. P., \& Wang, Y.-H. 1997, ApJ, 477, 905

Harlow, J. J. B., Pavlov, G. G., \& Halpern, J. R. 1998, AAS Meeting, 193, \#41.07

Helfand, D. J., Gotthelf, E. V., \& Halpern, J. P. 2001, ApJ, 556, 380

Hester, J. J., Mori, K., Burrows, D., et al. 2002, ApJ, 577, L49

Gaensler, B. M. 2005, ASpR, 35, 1116

Gaensler, B. M., Stappers, B. W., Frail, D. A., \& Johnston, S. 1998, ApJ, 499, L69

Jacchia, A., de Luca, F., Lazzaro, E., et al. 1999, A\&A, 347, 494

Jackson, M. S., Halpern, J. P., Gotthelf, E. V., \& Mattox, J. 2002, ApJ, 578, 935

Kargaltsev, O. Y., Pavlov, G. G., Zavlin, V. E., \& Romani, R. W. 2005, ApJ, 625, 307

Kassim, N., \& Lazio, T. J. 1999, ApJ, 527, L101

Kennel, C. F., \& Coroniti, F. V. 1984, ApJ, 283, 694

Kern, B., Martin, C., Mazin, B., \& Halpern, J. P. ApJ, 597, 1049

Komarova, V., Shibanov, Yu., Zharikov, S., et al. 2003, Pulsars, AXPs and SGRs observed with BeppoSAX and Other Observatories, Proceedings of the International Workshop held in Marsala, September 23-25, 2002, ed. G. Cusumano, E. Massaro, \& T. Mineo, 77

Komarova, et al. 2005, in preparation

Koptsevich, A. B., Pavlov, G. G., Zharikov, S. V., et al. 2001 (K2001), A\&A, 370, 1004

Koptsevich, A. B., Lundqvist, P., Serafimovich, N. I., Shibanov, Yu. A., \& Sollerman, J. 2003, A\&A, 400, 265

Kuiper, L., Hermsen, G., Cusumano, G., et al. 2001, A\&A, 378, 918

Kurt, V. G., Sokolov, V. V., Zharikov, S. V., Pavlov, G. G., \& Komberg, B.V. 1998, A\&A, 333, 547

Kurt, V. G., Komarova, V. N., Sokolov, V. V., et al. 2001, BSAO, 51, 21

Kuz'min, A. D., \& Losovskii, B. Ya. 1997, Astron. Lett., 23, 283

Landolt, A. U. 1992, AJ, 104, 340

Macomb, D. J., \& Gehrels, N. 1999, ApJS, 120, 335
Malofeev, V. M., \& Malov, O. I. 1997, Nature, 389, 697

Marshall, H. L., \& Schulz, N. S. 2002, ApJ, 574, 377

Martin, C., Halpern, J. P., \& Schiminovich, D. 1998, ApJ, 494, L211

Mignani, R. P., Caraveo, P. A., \& Bignami, G. F. 1998, ApJ, 332, L37

Mignani, R. P., De Luca, A., \& Caraveo, P. A. 2000, ApJ, 543, 318

Mignani, R. P., De Luca, A., Caraveo, P. A., \& Becker, W. 2002, ApJ, 580, L147

Mignani, R. P., De Luca, A., Kargaltsev, O., et al. 2003, ApJ, 594, 419

Mignani, R. P., \& Becker, W. 2004, AdSpR, 33, 616

Miyazaki, S., Sekiguchi, M., Imi, K., et al. 1998, in Optical Astronomical Instrumentation, ed. S. D’Odorico, Proc. SPIE, 3355,363

Monet, D., Levine, S., Canzian, B., et al. 2003, AJ, 125, 984

Nousek, J. A., Cowie, L. L., Hu, E., Lindblad, C. J., \& Garmire, G. P. 1981, ApJ, 248, 152

Pavlov, G. G., Stringfellow, G. S., \& Córdova, F. A. 1996, ApJ, 467, 370

Pavlov, G. G., Welty, A. D., \& Córdova, F. A. 1997, ApJ, 489, L75

Pavlov, G. G., Zavlin, V. E., Sanwal, D., et al. 2001, ApJ, 554, 189

Pavlov, G. G., Zavlin, V. E., \& Sanwal, D. 2002, Proc. of the 270, WE-Heraeus Seminar on Neutron Stars, Pulsars, and Supernova Remnants, 273 [arXiv: astro-ph/0206024]

Pellizza, L. J., Mignani, R. P., Grenier, I. A., \& Mirabel, I. F. 2005, A\&A, 435, 625

Ramanamurthy, P. V., Fichtel, C. E., Harding, A. K., et al. 1996, A\&AS, 120, 115

Romani, R. W., Cordes, J. M., \& Yadigaroglu, I.-A. 1997, ApJ, 484, L137

Romani, R. W., \& Ng, C.-Y. 2003, ApJ, 585, L141

Romani, R. W., Kargaltsev, O., \& Pavlov, G. G. 2005, ApJ, 627, 383

Schlegel, D. J., Finkbeiner, D. P., \& Davis, M. 1998, ApJ, 500, 525

Serafimovich, N. I., Shibanov, Yu. A., Lundqvist, P., \& Sollerman, J. 2004, A\&A, 425, 1041

Shearer, A., Redfern, R. M., Gorman, G., et al. 1997, ApJ, 487, L181

Shearer, A., Golden, A., Harfst, S., et al. 1998, A\&A, 335, L21

Shibanov, Yu. A., Koptsevich, A. B., Sollerman, J., \& Lundqvist, P. 2003, A\&A, 406, 645

Shibanov, Yu. A., Sollerman, J., Lundqvist, P., Gull, T., \& Lindler, D. 2005, A\&A, 440, 693

Shitov, Yu. P., \& Pugachev, V. D. 1998, New Astron., 3, 101

Sollerman, J. 2003, A\&A, 406, 639

Sollerman, J., Lundqvist, P., Lindler, D., et al. 2000, ApJ, 537, 861

Taylor, J. H., Manchester, R. N., \& Lyne, A. G. 1993, ApJS, 88, 529

Thorsett, S. E., Benjamin, R. A., Brisken, W., et al. 2003, ApJ, 592, L71

Vishwanath, P. R., Sathyanarayana, G. P., Ramanamurthy, P. V., \& Bhat, P. N. 1993, A\&A, 267, L5

Weisskopf, M. C., O’Dell, S. L., Paerels, F., et al. 2004, ApJ, 601, 1050

Yakovlev, D. G., \& Pethick, C. J. 2004, ARA\&A, 42, 169

Zavlin, V. E., \& Pavlov, G. G. 2004a, Mem. Soc. Astron. It., 75, 458

Zavlin, V. E., \& Pavlov, G. G. 2004b, ApJ, 616, 452

Zharikov, S. V., Shibanov, Yu. A., Koptsevich, A. B., et al. 2002, A\&A, 394, 633

Zharikov, S., Mennickent, R., Shibanov, Yu., et al. 2003, Pulsars, AXPs and SGRs observed with BeppoSAX and Other Observatories, Proc. of the International Workshop held in Marsala, September 23-25, 2002

Zharikov, S. V., Shibanov, Yu. A., Mennickent, R. E., et al. 2004, A\&A, 417, 1017

Zharikov, S. V., Shibanov, Yu. A., \& Komarova, V. N. 2005, AdSpR, in press [arXiv: astro-ph/0410152] 